\title{
A quantitative point-of-care test for periodontal and dental peri-implant diseases
}

\author{
Timo Sorsa, Dirk Gieselmann, Nicole B. Arweiler and Marcela Hernández
}

In their recent Primer article (Periodontal diseases. Nat. Rev. Dis. Primers 3, 17038 (2017)) ${ }^{1}$, Denis F. Kinane and colleagues provide an important and comprehensive update on the pathogenesis, diagnosis, management and strategies of prevention of periodontal and peri-implant diseases. In this context, and in view of the latest advances in translational research on periodontal and peri-implant disease biomarkers, quantitative point-of-care (POC) technologies are emerging as new tools to target periodontitis and peri-implantitis. In particular, these technologies could help to pinpoint the crucial transition of gingivitis or subclinical periodontitis without clinical or radiographic manifestations to active periodontal disease, with progressive deepened pockets and attachment loss ${ }^{2}$.

As stated in the Primer, the diagnosis of periodontal and peri-implant diseases is mainly based on the clinical measurements of pocket depth, attachment loss and bleeding on probing, together with radiographic examination. These diagnostic procedures can assess only past tissue destruction and do not provide any information about the current disease status or future progression. The accurate assessment of disease progression by conventional clinical and radiographic means is further complicated by the episodic progression of the disease course. Neutrophil collagenase, also known as matrix metalloproteinase 8 (MMP8), has been identified as a major collagenolytic enzyme that causes periodontal tissue destruction in periodontitis and peri-implantitis, as well as being found in gingival crevicular fluid, peri-implant sulcular fluid, mouth rinse and saliva ${ }^{2}$. A key characteristic of active periodontal disease is the pathological elevation of the levels and activation of MMP8 in oral fluids, which was not covered in extensive detail in the Primer.

Indeed, a quantitative POC activated MMP8 (aMMP8) oral fluid test has been repeatedly and independently validated in Finland, Germany, Nigeria, Turkey, the Netherlands and the United States to successfully screen susceptible sites and patients, differentiate active and inactive periodontal sites, predict the future disease progression and monitor treatment response and maintenance therapy ${ }^{3-8}$, with a diagnostic sensitivity and specificity of $76-83 \%$ and $96 \%$, respectively, and a turnaround time of 5-7 minutes. Furthermore, the test can identify initial periodontitis in genetically predisposed adolescents ${ }^{5}$. Thus, the aMMP8 test is effective in both adolescent and adult populations ${ }^{3,4}$. The predictive value of the test lies in its ability to detect subclinical periodontitis before clinical or X-ray manifestations, as the test is positive (that is, aMMP8 level in oral fluids is elevated) ahead of active periodontal disease $\mathrm{e}^{9-13}$. The test is, therefore, very suitable for monitoring disease progression and tailoring preventive and therapeutic measures $^{14}$. The aMMP8 test is inexpensive, easy to use (the results are automated and, therefore, independent of the practitioner's experience) and currently available for routine use by dental and medical professionals linking these disciplines ${ }^{3-5,8,15}$. Finally, we agree with the authors of the Primer that additional work, especially on the prognostic value of biomarkers in periodontal and peri-implant diseases, is still required.

Timo Sorsa is at the Department of Oral and Maxillofacial Diseases, University of Helsinki and Helsinki University Hospital, Helsinki, Finland; and at the Department of Dental Medicine, Karolinska Institutet, Huddinge, Sweden.

Dirk Gieselmann is at the Institute of Molecular Dentistry, Solingen, Germany.

Nicole B. Arweiler is at the Department of Periodontology, Philipps University of Marburg, Marburg, Germany.

Marcela Hernández is at the Laboratory of Periodontal Biology and Department of Oral Pathology and Medicine, Faculty of Dentistry, Universidad de Chile, Av. Sergio Livingstone Polhammer 943, Independencia, Santiago 8380492, Chile; and at the Dentistry Unit, Faculty of Health Sciences, Universidad Autónoma de Chile, El Llano Subercaseaux 2801, San Miguel Santiago 8910060, Chile.

Correspondence to M.H. mhernandezrios@gmail.com

doi: $10.1038 /$ nrdp. 2017.69 Published online 14 Sep 2017

1. Kinane, D. F., Stathopoulou, P. G. \& Papapanou, P. N. Periodontal diseases. Nat. Rev. Dis. Primers 3, 17038 (2017).

2. Sorsa, T. et al. Analysis of matrix metalloproteinases, especially MMP-8, in gingival crevicular fluid, mouthrinse and saliva for monitoring periodontal diseases. Periodontol. 2000 70, 142-163 (2016).

3. Nwhator, S. O. et al. Clinical correlates of a lateral-flow immunoassay oral risk indicator. J. Periodontol. $\mathbf{8 5}$, 188-194 (2014).

4. Heikkinen, A. M. et al. Pilot study on oral health status as assessed by an active matrix metalloproteinase- 8 chairside mouthrinse test in adolescents. J. Periodontol. 87, 36-40 (2016)

5. Heikkinen, A. M. et al. Pilot study on the genetic background of an active matrix metalloproteinase- 8 test in Finnish adolescents. J. Periodontol. 88, 464-472 (2017).

6. Lorenz, K. et al. Evaluation of a novel point-of-care test for active matrix metalloproteinase-8: agreement between qualitative and quantitative measurements and relation to periodontal inflammation. J. Periodontal. Res. 52, 277-284 (2017).

7. Johnson, N. et al. Rapid assessment of salivary MMP-8 and periodontal disease using lateral flow immunoassay. Oral. Dis. 22, 681-687 (2016).

8. Izadi Borujeni, S., Mayer, M. \& Eickholz, P. Activated matrix metalloproteinase- 8 in saliva as diagnostic test for periodontal disease? A case-control study. Med. Microbiol. Immunol. 204, 665-672 (2015).

9. Sorsa, T. et al. Matrix metalloproteinases: contribution to pathogenesis, diagnosis and treatment of periodontal inflammation. Ann. Med. 38, 306-321 (2006).

10. Lee, W., Aitken, S., Sodek, J. \& McCulloch, C. A Evidence of a direct relationship between neutrophil collagenase activity and periodontal tissue destruction in vivo: role of active enzyme in human periodontitis. J. Periodontal. Res. 30, 23-33 (1995).

11. Mancini, S. et al. Assessment of a novel screening test for neutrophil collagenase activity in the diagnosis of periodontal diseases. J. Periodontol. 70, 1292-1302 (1999).

12. Leppilahti, J. M., Kallio, M. A., Tervahartiala, T., Sorsa, T. \& Mantyla, P. Gingival crevicular fluid matrix metalloproteinase-8 levels predict treatment outcome among smokers with chronic periodontitis. J. Periodontol. 85, 250-260 (2014).

13. Leppilahti, J. M. et al. The utility of gingival crevicular fluid matrix metalloproteinase-8 response patterns in prediction of site-level clinical treatment outcome. J. Periodontol. 86, 777-787 (2015).

14. Axelsson, P., Nystrom, B. \& Lindhe, J. The long-term effect of a plaque control program on tooth mortality, caries and periodontal disease in adults. Results after 30 years of maintenance. J. Clin. Periodontol. 31, 749-757 (2004)

15. Sorsa, T. et al. Collagenase-2 (MMP-8) as a point-of-care biomarker in periodontitis and cardiovascular diseases. Therapeutic response to non-antimicrobial properties of tetracyclines. Pharmacol. Res. 63, 108-113 (2011).

Competing interests

T. S. is an inventor of US patents 5652223,5736341 , 5866432 and 6143476 . All other authors declare no competing interests.

How to cite this article

Sorsa, T., Gieselmann, D., Arweiler, N. B. \& Hernández, M. A quantitative point-of-care test for periodontal and dental peri-implant diseases. Nat. Rev. Dis. Primers 3, 17069 (2017). 